\title{
Modulation of primary orthostatic tremor by magnetic stimulation over the motor cortex
}

\author{
C H Tsai, J G Semmler, T E Kimber, G Thickbroom, R Stell, F L Mastaglia, \\ P D Thompson
}

\begin{abstract}
Objectives-To study the role of corticomotor neuronal pathways in primary orthostatic tremor.

Methods-Transcranial magnetic stimuli at an intensity $10 \%$ above the resting motor threshold were delivered over the leg motor cortex in two patients with primary orthostatic tremor while standing still. Electromyographic responses in both tibialis anterior muscles were recorded after 20 stimuli given randomly at intervals of 120 to 180 seconds. Differences between predicted and actual times of occurrence of tremor bursts after the stimuli were used to calculate a resetting index, with a value of 0 representing no resetting and a value of 1 representing complete resetting.

Results-Transcranial magnetic stimulation evoked EMG responses in both tibialis anterior muscles, followed by transient suppression of tremor before reappearance of rhythmic EMG activity. Analysis of the timing of tremor bursts from EMG recordings before and after the magnetic stimuli disclosed that the phase of orthostatic tremor could be reset by brain stimulation (mean resetting indices 0.93 and 0.82 ).

Conclusion-The results suggest that a central oscillator, involving the motor cortex, has a crucial role in either the generation or modulation of orthostatic tremor.
\end{abstract}

(F Neurol Neurosurg Psychiatry 1998;64:33-36)

Keywords: orthostatic tremor; tremor resetting; transcranial magnetic stimulation

Primary orthostatic tremor is thought to be generated by central oscillators without modification by peripheral inputs. ${ }^{12}$ Whether these oscillators involve cortical circuits is not known. The role of the motor cortex in orthostatic tremor was investigated in this study using the technique of transcranial magnetic stimulation. If the cortex has an important role in orthostatic tremor, brain stimulation should reset the tremor, as can be demonstrated, for example, in parkinsonian tremor. ${ }^{34}$ If the tremor is unresponsive to brain stimulation, then a subcortical origin for the tremor would be more likely.

\section{Patients and methods}

PATIENTS

Two female patients aged 58 and 68 years with a typical history and physical signs of orthostatic tremor of six and 30 years duration respectively were studied. Both patients gave informed consent before the study. Electromyographic recordings of tremor in leg muscles disclosed $16 \mathrm{~Hz}$ tremor during standing. Tremor was associated with unsteadiness. No other neurological abnormalities were evident.

METHODS

Surface EMG recordings from both tibialis anterior muscles were made using silver/silver chloride electrodes taped $3 \mathrm{~cm}$ apart over the muscles. The EMG signals were amplified (MacLab/8 (AD Instruments, NSW, Australia)) bandpass filtered $(10 \mathrm{~Hz}$ to $2.5 \mathrm{kHz})$, digitised (sampling rate $1 \mathrm{kHz}$ per channel), and stored on computer. EMG activity was recorded for one second before and 1.5 seconds after the transcranial magnetic stimulus. Each trial was stored on computer and later retrieved, full wave rectified, and digitally smoothed for off line measurement.

The motor cortex was stimulated using a MAGSTIM 200 (Whitland, Dyfed UK). A double cone coil (inner diameter $9.6 \mathrm{~cm}$; outer diameter $12.5 \mathrm{~cm}$ ), designed for stimulation of the leg area of the motor cortex, was placed over the vertex. The stimulus intensity was set at $10 \%$ of stimulator output above the threshold for eliciting EMG responses in relaxed tibialis anterior muscles. Cortical stimuli were given randomly at intervals of 120 to 180 seconds. Patients were stimulated while standing still, in a position resulting in typical tremor of the legs. Between stimuli, they were seated comfortably.

The rectified tibialis anterior EMG of each trial was averaged by computer (fig 1 ). Because magnetic stimuli were given at random times within the tremor cycle, this had the effect of "averaging out" the EMG bursts preceding the stimulus, so producing a relatively flat average rectified EMG trace of prestimulus muscle activity. If magnetic stimuli had no effect on the tremor, there would be a level trace in the period after stimulation as well. However, if the timing of EMG bursts in the tibialis anterior muscle was modulated in a consistent manner (for example, phase reset) by magnetic brain stimuli, then the average rectified EMG trace after stimulation will show such modulation.

To quantify the effect of magnetic brain stimuli on tremor, we employed the "resetting index" (RI), ${ }^{36}$ which was calculated in the following manner. From the single raw EMG 
traces, the average period between tremor bursts (average cycle length) was calculated for the 10 EMG bursts preceding the magnetic stimulus. The timing of the magnetic stimulus relative to the timing of the last EMG burst before the magnetic stimulus was then expressed as a proportion of the average cycle length (\% cycle length). Predicted timings for the subsequent 10 tremor bursts (had there not been a magnetic stimulus) were then calculated based on the timing of the last burst before the stimulus and the average cycle length. The actual timing of 10 tremor bursts after stimulation was measured. A graph was then plotted of timing of the magnetic stimulus in the ongoing tremor cycle against the actual-expected timing of the subsequent 10 EMG bursts. For each EMG burst after the magnetic stimulus, a linear regression line was derived. The slope of the lines give an indication of the resetting that has occurred; a slope of 0 implies no resetting, whereas a slope of 1 implies complete resetting. The RI was calculated by taking the average slope of the regression lines for the $10 \mathrm{EMG}$ bursts after the magnetic stimulation. ${ }^{4}$

In many trials it was often difficult to decide whether the initial EMG activity after magnetic stimulation was due to rebound during the silent period or resumption of orthostatic tremor. To account for this, the first tremor burst after the magnetic stimulus in each raw trace was defined by the timing of the first EMG burst of tremor in the averaged record. Results were obtained from a total of 20 trials in each patient.

Statistical analysis of group data was performed with repeated measure analysis of variance (ANOVA) and paired Student's $t$ tests as appropriate. Correlation was performed by Pearson's correlation test. For all analysis, significance was reported for $\mathrm{P}<0.05$.

\section{Results}

In all records, each magnetic stimulus resulted in a short latency motor response, followed by a transient suppression of the EMG activity (silent period), before rhythmic EMG activity resumed at about $160 \mathrm{~ms}$ after the stimulus. Figure 1 illustrates the effect of averaging all individual sweeps, aligned according to the timing of occurrence of the magnetic stimulus. The occurrence of oscillatory EMG activity in the averaged EMG signal after the stimulus indicates that the tremor phase has been reset and become time locked to the stimulus. The calculated RI in case 1 was 0.87 for the right leg and 0.99 for the left leg (fig 1, upper two traces), and in case 2 it was 0.86 for the right leg and 0.79 for the left leg (fig 1, lower two traces).

Figure 2 shows the time course of tremor behaviour after magnetic stimulation. In all 20
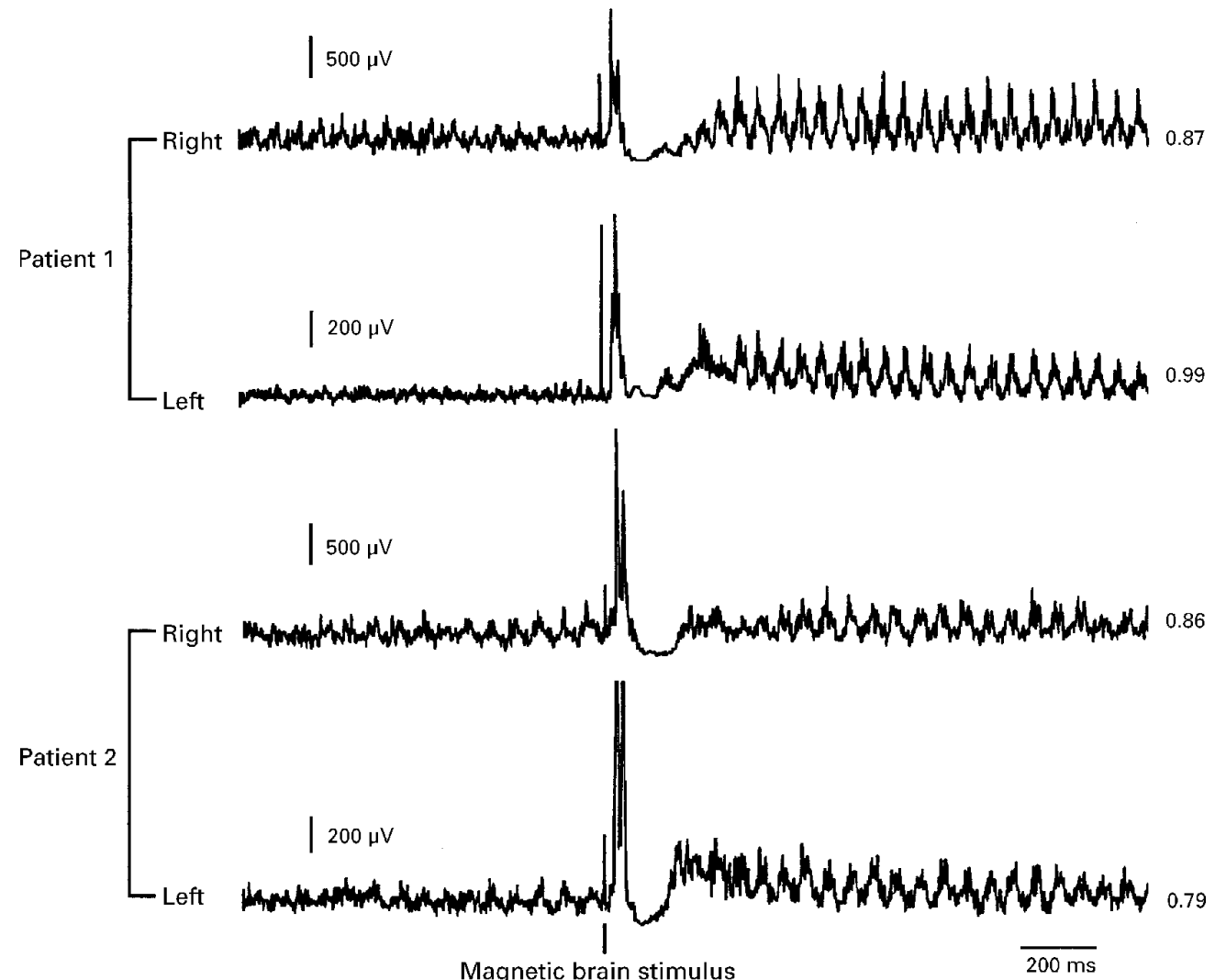

Figure 1 Modulation of bilateral orthostatic tremor by magnetic stimulation over the leg motor cortex. Traces represent the average of 20 rectified single trials. The individual resetting index is shown to the right of each trace. The upper two traces are recordings from case 1 and the lower traces are from case 2. The average traces in both legs show modulations of rhythmic EMG activity after transcranial magnetic stimulation and a change in timing of the tremor bursts which have become time locked to the stimulus. 

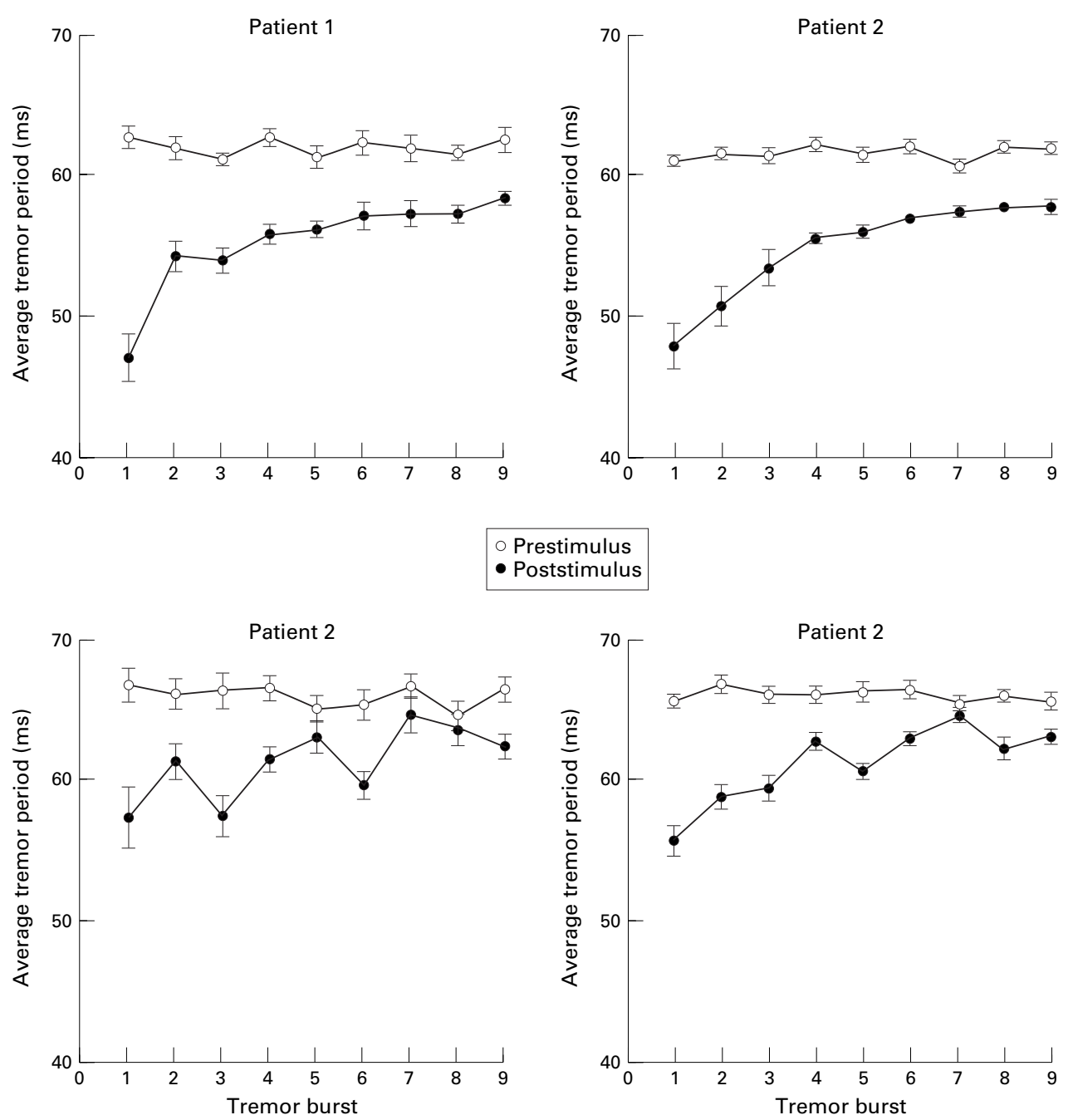

Prestimulus

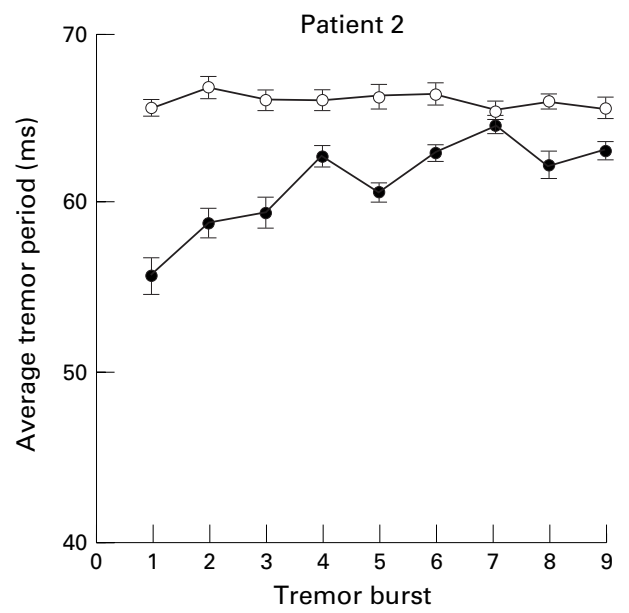

Figure 2 Time course of orthostatic tremor period evolution before and after magnetic stimulation from the first (1) to the ninth burst (9) before and after stimulation. The initial reburst tremor periods were around $70 \%-80 \%$ of the prestimulus levels, which then returned gradually toward the ongoing levels. Data are the means (SEM) of the respective tremor period from 20 trials.

trials, the average cycle length of the initial 10 tremor bursts after the stimulus tended to be shorter than those of the prestimulus tremor. On resumption of tremor, the average cycle length was about $70 \%-80 \%$ of the prestimulus levels, and gradually returned to the prestimulus tremor period. Poststimulus cycle lengths were shorter by an average of $6.4 \mathrm{~ms}$ in case 1 and $4.8 \mathrm{~ms}$ in case 2 ( $t$ tests all $\mathrm{P}<0.01)$. Repeated measures ANOVA between prestimulus and poststimulus average cycle lengths in both cases were significantly different (right, $\mathrm{P}<0.01$; left, $\mathrm{P}<0.01$ ).

To investigate whether the tremor bursts on both legs were synchronised before stimulation, the timing of the magnetic stimulus within a tremor cycle was measured and expressed as a percentage of the total average cycle length (\% cycle length; fig 3 ) on both sides. The degree of synchrony was estimated by a comparison of the \% cycle length for right and left legs. The results showed a high correlation in $\%$ cycle length between left and right legs in both patients (case 1: correlation coefficient $0.91, \mathrm{P}<0.001$; case 2 : correlation coefficient
$0.97, \mathrm{P}<0.001)$ and suggested a synchronisation of tremor bursts in both legs before magnetic stimulation (fig 3).

\section{Discussion}

This study has shown that transcranial magnetic stimulation can reset and modulate orthostatic tremor of the legs. As the volleys evoked by magnetic stimulation were conveyed from the motor cortex to the spinal cord, where spinal motor neurons were discharged, resetting could occur at each of these levels. Electrical stimulation of the peroneal nerve is ineffective in modulating or resetting orthostatic tremor bursts in the tibialis anterior, making it unlikely that a peripheral feedback mechanism accounts for the resetting of this tremor after brain stimulation (unpublished observations). ${ }^{12}$ Furthermore, the $16 \mathrm{~Hz}$ tremor found in primary orthostatic tremor greatly exceeds that expected from oscillations in an overactive spinal reflex arc. Accordingly, central circuits must have a crucial role in the tremor resetting. Several features suggest that central factors might be important. Firstly, the 

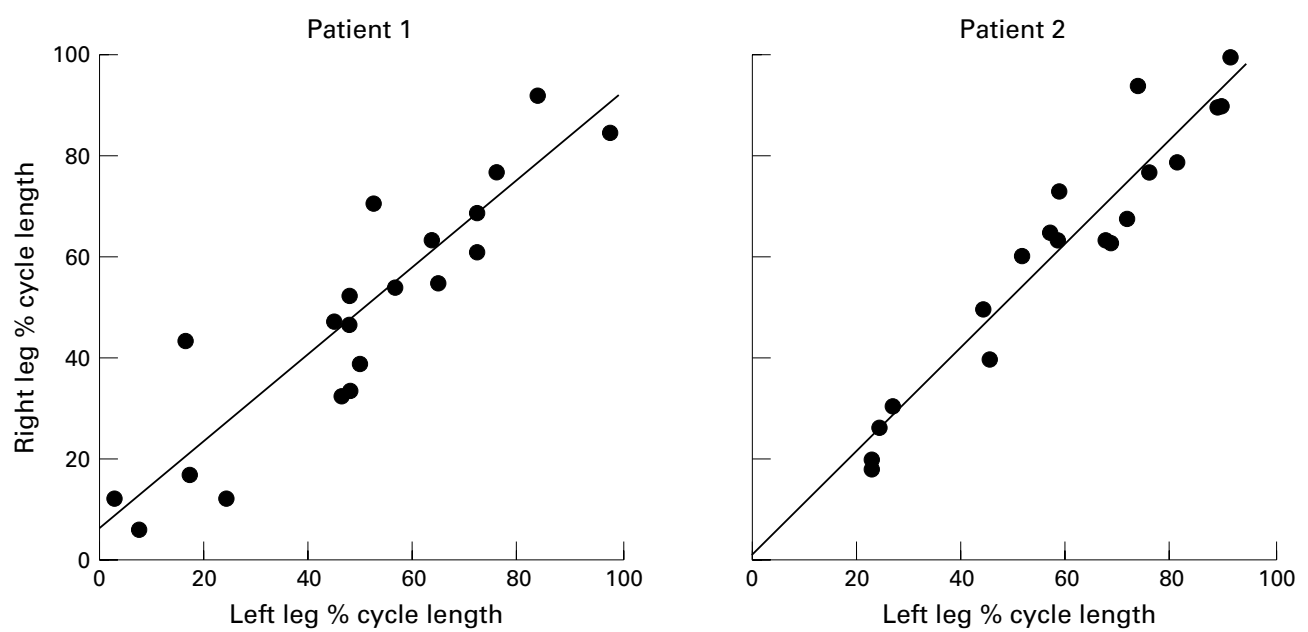

Figure 3 Relation between the \% cycle length of tremor bursts in the left and right legs of two patients with primary orthostatic tremor. The \% cycle length indicates the timing of the tremor burst preceding the magnetic stimulus expressed as a percentage of the total tremor cycle. The linear regression correlation coefficient for case 1 was 0.91 and case 2 was 0.97 (both $P<0.001$ ). The strong correlation between the \% cycle length in left and right legs in both cases indicates a high degree of synchronisation of the tremor bursts in left and right legs.

phase of tremor was highly synchronised in both legs (fig 3). This finding was compatible with a previous single motor unit cross correlation study in primary orthostatic tremor, in which close linkage of motor unit discharges was detected between both tibialis anterior muscles, strengthening the central hypothesis. ${ }^{2}$ Secondly, in most patients with orthostatic tremor, including the present cases, a tremor of similar frequency in proximal upper limb muscles is usually present when patients stand with their arms outstretched, either in front of or behind their bodies. ${ }^{1279}$ If central circuits are involved in the genesis of the primary orthostatic tremor, how does this fit with the current understanding of pathophysiological mechanisms of tremor? Recently, a "two loop" hypothesis has been proposed as a model for parkinsonian tremor. ${ }^{10}$ In this model, the intrinsic loop, involving striatothalamocorticostriatal circuits, interacts with an extrinsic loop in which short latency reafferent input to the ventralis intermedius nucleus of the thalamus (via cerebellar pathways) projects to the motor cortex and may serve to stabilise oscillation in the intrinsic loop. In the case of parkinsonian tremor, it was postulated that the extrinsic loop was modified by magnetic cortical stimulation or mechanical perturbation, culminating in tremor resetting or modulation. ${ }^{10}$ Interruption of a cortical projection is likely to account for resetting of orthostatic tremor by magnetic brain stimulation. Subcortical circuits also seem to be involved in orthostatic tremor. A recent PET study of primary orthostatic tremor showed that, when patients maintained a posture with their right upper limb outstretched, abnormal bilateral cerebellar and contralateral lentiform and thalamic activation were detected. ${ }^{11}$

The period of the tremor after stimulation was significantly shorter than that before stimulation, particularly in the early stages of tremor reappearance (fig 2). It is of interest that a shortening of the tremor period was also reported in parkinsonian postural tremor but not in essential or mimicked tremors after transcranial magnetic stimulation. ${ }^{5}$ The phenomena suggest the pathophysiological mechanisms underlying primary orthostatic tremor and parkinsonian tremor are different from those responsible for essential and mimicked tremors and indicate a prominent role of the motor cortex in their generation or modulation.

1 Thompson PD, Rothwell JC, Day BL, Berardelli A, Dick JPR, Kachi T, Marsden CD. The physiology of orthostatic JPR, Kachi T, Marsden CD. The physi
tremor. Arch Neurol 1986;43:584-7.

2 Britton TC, Thompson PD, van der Kamp W, Rothwell JC, Day BL, Findley LJ, Marsden CD. Primary orthostatic tremor: further observation in six cases. $\mathcal{F}$ Neurol 1992;239: 209-17.

3 Lee RG, Stein RB. Resetting of tremor by mechanical perturbations: a comparison of essential tremor and parkinsonian tremor. Ann Neurol 1981;10:523-31.

4 Britton TC, Thompson PD, Day BL, Rothwell JC, Findley LJ, Marsden CD. Resetting of postural tremors at the wrist with mechanical stretches in Parkinson's disease, essential tremor, and normal subjects mimicking tremor. Ann Neurol tremor, and normal

5 Britton TC, Thompson PD, Day BL, Rothwell JC, Findley LJ, Marsden CD. Modulation of postural wrist tremors by magnetic stimulation of the motor cortex in patients with Parkinson's disease or essential tremor and in normal subjects mimicking tremor. Ann Neurol 1993;33:473-9.

6 Pascual-Leone A, Valls-Sole J, Toro C, Wassermann EM, Hallett M. Resetting of essential tremor and postural tremor in Parkinson's disease with transcrainal magnetic tremor in Parkinson's disease with transcrion. Muscle Nerve 1994;17:800-7.

7 Gabellini AS, Martinelli P, Gulli MR, Ambrosetto G, Ciucci Gabellini AS, Martinelli P, Gulli MR, Ambrosetto G, Ciucci
G, Lugaresi E. Orthostatic tremor: essential and symptomatic cases. Acta Neurol Scand 1990;81:113-7.

8 Papa SM, Gershanik OS. Orthostatic tremor: an essential tremor variant. Mov Disord 1988;3:97-108.

9 Uncini A, Onofrj M, Basciani M, Cutarella R, Gambi D. Orthostatic tremor: report of two cases and an electrophysiological study. Acta Neurol Scand 1989;79:119-22.

10 Volkmann J, Joliot M, Mogilner A, Ioannides AA, Lado F, Fazzini E, et al. Central motor loop oscillations in parkinsonian resting tremor revealed by magnetoencephalography. nian resting tremor revealed
Neurology 1996;46:1359-70.

11 Wills AJ, Thompson PD, Findley LJ, Brooks DJ. A positron emission tomography study of primary orthostatic tremor. Neurology 1996;46:747-52. 